Skin cancer is the most frequently occurring neoplasm in the world. In the last several decades its increasing occurrence has been observed. The basic method of treatment of skin cancers is surgical resection of the lesion. There are numerous alternative methods to resection of the lesion, such as cryosurgery, radiotherapy, photodynamic therapy, or pharmacological preparations (5-fluorouracil, 5\% imiquimod - Aldara). Imiquimod is one of the more recent forms of treatment of skin cancers. Its mechanism is not fully known, though above all the activation of the immune system is postulated. This study presents a group of $4 \mathrm{pa}$ tients who were offered treatment with imiquimod preparation as an alternative to surgical resection of the lesion. Complete regression of the lesions was observed in all of the patients and during the 12-26 months of observation no relapse of the neoplastic disease was noted. No significant adverse reactions were observed during treatment. It seems that the chief field of application of imiquimod may be skin lesions in locations difficult for surgical dressing, extensive wounds and in a group of elderly patients with numerous con comitant diseases, where all the treatment can be carried out at home.

Key words: imiquimod, Aldara, skin cancer.

\section{Imiquimod as an alternative to surgery in the treatment of skin cancers - experience with the first group of patients}

\author{
Dawid Murawa ${ }^{12}$, Karol Połom ${ }^{12}$, Paweł Murawa
}

${ }^{1} 1^{\text {st }}$ Department of Oncological and General Surgery, Greater Poland Cancer Centre, Poznań

2Operative Department, MedPolonia Private Hospital, Poznań

\section{Introduction}

In the last decade a considerable increase in the frequency of occurrence of skin cancer has been observed. According to the WHO it is the every third newly diagnosed tumour. Basal cell carcinoma (BCC) and squamous cell carcinoma (SCC) make up the vast majority (more than $80 \%$ of skin cancers) [1]. For example, the frequency of occurrence of skin cancers is higher than that of mammary gland cancer, lung cancer and colorectal cancer altogether. The notable increase in the incidence of skin cancers in recent decades has been related to several factors: increased exposure to sunlight (the popularity of tourism to tropical countries), changes in fashion (uncovered parts of the body), extended life expectancy and the destruction of the ozone layer. The rate of increase is estimated at $10-15 \%$ per decade. Basal cell carcinoma is a slowly growing tumour, which comes from the cells of the basal layer of the epidermis. The lesion is locally malignant and it has a low metastatic potential ranging between 0.0028 and $0.1 \%$. SCC also derives from dysplastic epidermal cells. In contrast to BCC, SCC is preceded by preneoplastic lesions, especially by actinic keratosis. Also, it has a higher metastatic potential, reaching even $11 \%$ for lesions located on the lips and ears. In contrast to SCC, BCC can more often be found in patients with a reduced immune response (e.g. patients during immunosuppression after transplantations of organs). In spite of all those differences, both cancers can be found in patients with: fair complexion (skin phototypes I and II according to Fitzpatrick), burning tendency, freckles after sun exposure, and personal or family history of skin cancers. It is also worth mentioning that both types of neoplasms occur more often in patients who have undergone radiotherapy.

In spite of the fact that surgery remains the gold standard of skin cancer treatment, recently more and more frequently new treatment methods, which are non-surgical, have been suggested. This particularly concerns patients who cannot be qualified or who do not agree to surgery, who have extensive lesions or lesions that are in a difficult location. Thus they become a challenge for surgeons and they frequently involve the need to apply extensive plastic surgery. It also worth mentioning that such methods of skin cancer treatment as the application of Aldara (imiquimod) preparation, 5-fluorouracil or photodynamic therapy result in better cosmetic effects and thus they improve the quality of life. Imiquimod cream seems to be the most promising alternative to surgical treatment. Due to its small size and non-polar nature it easily penetrates the epidermis, thus being a perfect means of treatment of preneoplastic and neoplastic skin lesions. Imiquimod acts anti-neoplastically through different mechanisms. Three main ways of its action have been demonstrated. First of all, it leads to the production of cytokines, chemokines and other mediators of the local inflammatory response [2]. Second, imiquimod inten- 

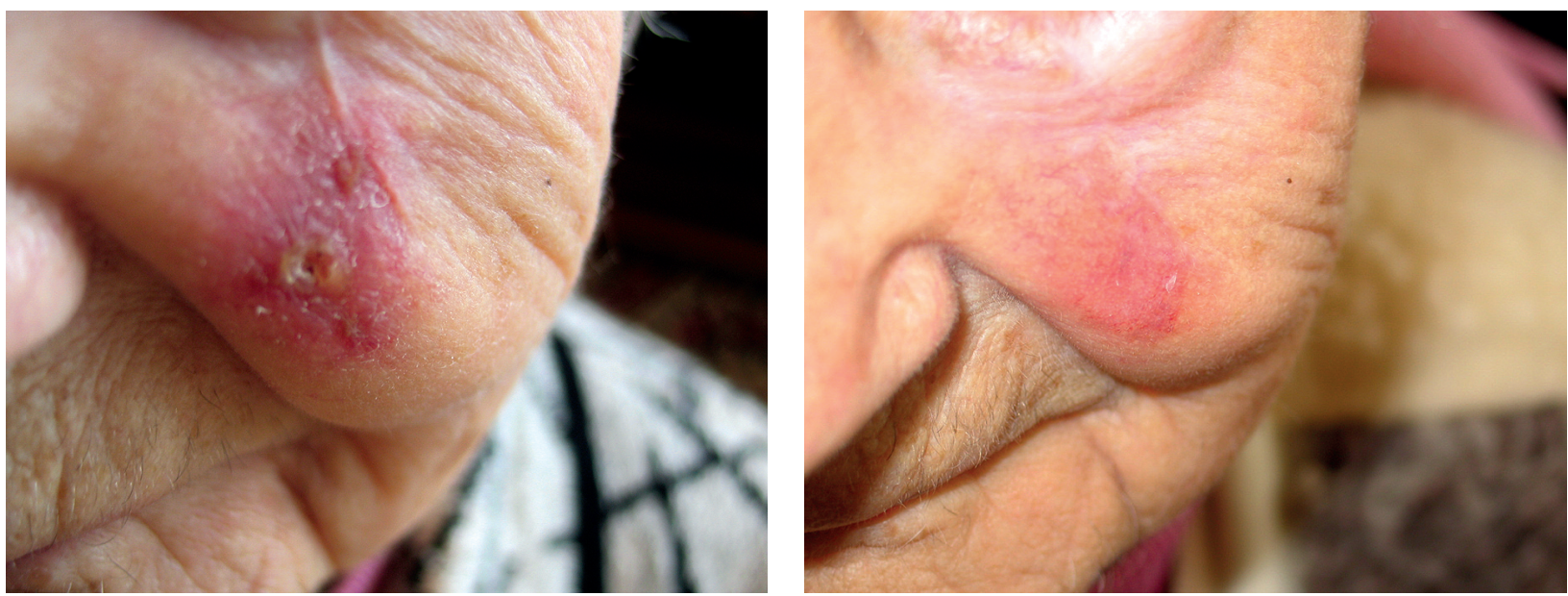

Fig. 1. Patient, aged 102 years, treated due to a relapse of squamous cell carcinoma of the skin on the left cheek. The state before and 2 weeks following the end of therapy

sifies the inflammatory response. Third, a high concentration of the drug induces apoptosis of neoplastic cells $[3,4]$. In the USA imiquimod as a $5 \%$ cream has been approved by the FDA for treatment of genital warts in the genital and perianal area, basal cell carcinoma in patients where the lesion does not exceed $2 \mathrm{~cm}$ in diameter on the torso, neck and limbs (hands and feet excluded), and clinically typical actinic keratosis on the face or skin of a hairy head. In Europe the indications are similar, except the limitations concerning the treatment of basal cell cancer depending on the location and size of the lesion [5].

\section{Material and methods}

The group consisted of 4 patients ( 3 men and 1 woman) aged from 78 to 102 years with facial skin cancer diagnosed ( 2 patients with basal cell carcinoma and 2 patients with squamous cell carcinoma). Due to their general state of health and considerable ambulatory difficulties, after consultations with their families 2 of the patients were qualified for treatment by means of $5 \%$ imiquimod cream. After earlier operations of numerous lesions the other 2 patients decided to continue therapy of residual focuses with this method.

All the patients were treated in a 6-week system: the cream was administered for 5 days followed by a 2-day interval. One patient with squamous cell carcinoma received another course after 2 months following the end of the first course due to a residual focus. All the patients rubbed a thin layer of the cream into their skin with the lesion, leaving a $1 \mathrm{~cm}$ margin around. The application was recommended in late hours, immediately before sleep.

\section{Results}

No significant adverse reactions were observed in any of the treated patients during the therapy. One patient experienced slightly higher temperature during the first week of therapy. Complete remission of the lesions was noted in all of the patients. However, in 1 patient with squamous cell carcinoma remission was observed after the second course, whereas after the first course the lesion was reduced by about $40-60 \%$. During the therapy the characteristic feature was massive inflammatory reaction of the skin with periodic oozing in the place of drug application (all the patients were informed about the problem before the therapy). There was a regression of the process in all the patients in 3-4 weeks following the end of the therapy, leaving no discolouration of the skin. During the 12-26 months of the observation period no signs of local relapse were noted (fig. 1-3).

\section{Discussion}

According to the described observations of complete curability at the level of 69-100\% for superficial basal cell carcinoma (sBCC) and $42-100 \%$ for nodular basal cell carcinoma (nBCC), imiquimod may become an effective alternative to surgical treatment [6]. Nevertheless, the rules of therapy with this drug remain under discussion. In a study with randomisation the drug was administered for 6 weeks once a day 7 days a week, or once a day 3 days a week, or twice a day 3 days a week [7]. Six weeks after the end of the therapy complete remission of the lesion was observed in $88 \%$, $70 \%$ and $73 \%$ of the patients respectively. These results clearly indicate better efficacy of the therapy lasting 7 days a week rather than shorter applications, between which there are no significant differences in curability. In further study a longer period of drug administration was subjected to analysis -12 weeks [8]. Three groups of patients were selected: the first group received the drug once a day 7 days a week, the second group once a day 5 days a week, and the third group once a day 3 days a week. Histologically confirmed regression of the lesions was observed in $87 \%, 81 \%$ and $51 \%$ of the patients respectively. This study emphasised that in comparison with the 6 -week method of drug administration the 12-week period seems to be more effective. However, it is worth mentioning that along with increased efficacy of the therapy an increased number of adverse side effects was observed. 

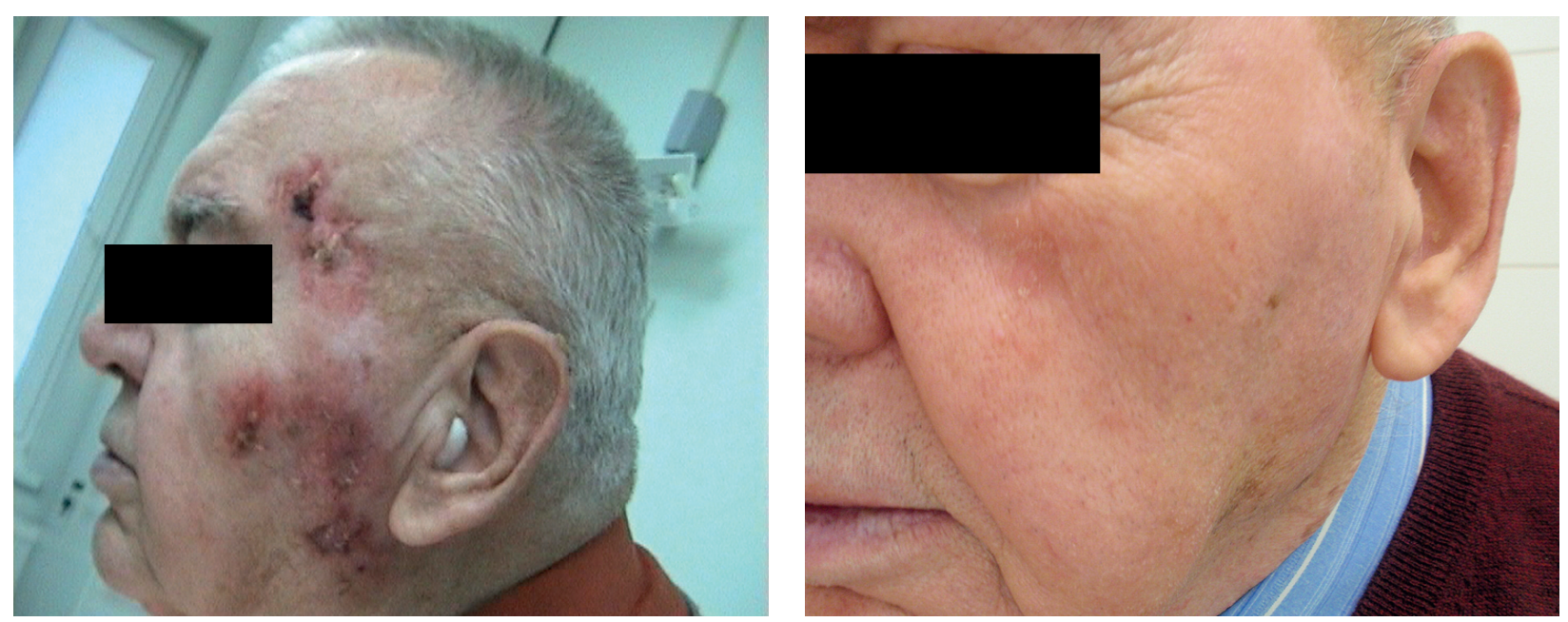

Fig. 2. Patient, aged 78 years, treated due to multifocal basal cell carcinoma of the skin on the left half of the face. The state before and 5 weeks after therapy
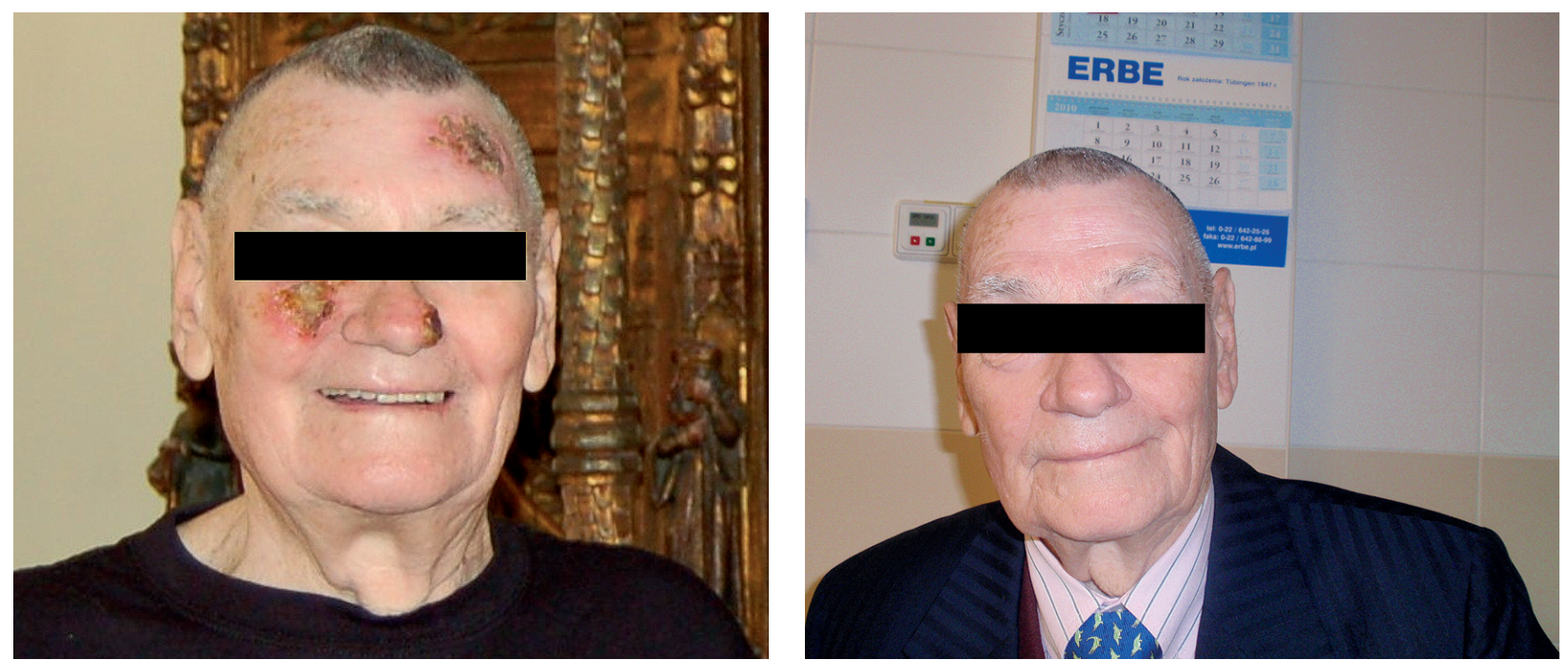

Fig. 3. Patient, aged 85 years, treated due to multifocal basal cell carcinoma of the facial skin. The state during treatment (middle of therapy) and 6 weeks following the end of therapy (Prof. S. Paszyc agreed to show the patients' faces)

In the study where the efficacy of application of $5 \%$ imiquimod was assessed in a 12 -week method of BCC treatment once a day 3 times a week in a group of 75 patients, efficacy of the drug was observed in 70 of them (93.3\%). Local relapse was observed in $2.9 \%$ of the patients during the observation period lasting up to 34 months (23 months on average). Local reaction was observed in $67.3 \%$ of the patients [9]. Lymphadenopathy was not diagnosed in any of the patients. Most accessory symptoms were limited to the place of imiquimod administration and the most frequently observed reactions were itch and pain (67.3\%) and reddening (63.3\%). Other observed side effects related to the application of imiquimod were swelling, anaesthesia, ulceration and burning sensation. General accessory symptoms and lymphadenopathy are rather rare; nevertheless they can be observed [10]. Other symptoms quoted in the literature include reduced haematological values in periph- eral blood (WBC, RBC, PLT). Serious symptoms resembling influenza, very rare skin reactions such as erythema multiforme, Stevens-Johnson syndrome and lupus erythematosus were also described.

Histological remission is surprisingly high, especially when compared with other methods of non-surgical treatment of BCC. However, the data reflecting the frequency of relapse are not so optimistic and it reaches from $2.9 \%$ to as much as $16 \%$ in a period of observation exceeding 2 years $[9,11]$. There results from this an image of high frequency local relapse when imiquimod is applied in monotherapy, especially in comparison with surgical treatment, where the frequency of local relapse is $3-8 \%$ of patients. Therefore imiquimod is recommended to be used in the treatment of BCC as an additional form of therapy in combination with other methods. In a randomised study of the second phase with a double-blind trial in which 5\% imiquimod was admin- 
istered 5 days a week for 2-6 weeks before further treatment of sBCC and $\mathrm{nBCC}$ by means of Mohs micrographic surgery [12], considerable reduction of the lesion was observed, which resulted in better cosmetic effects after surgical resection. In another study 2 groups of patients subjected to curettage and electrodissection received 5\% imiquimod or cream with placebo for 1 month following the surgery [13]. In the group treated with imiquimod better effects of the therapy were observed and a better cosmetic effect in comparison with those treated by means of curettage and cream with placebo.

One of the most important advantages of using imiquimod is its possibility to be applied by the patient at home. The frequency and optimal time of administration of the drug are a problem which has not been solved yet. The most frequently recommended dosage is 5 days a week for 6 weeks (the manufacturer's recommendation). We treated our patients with this system.

Imiquimod seems to be an efficacious drug in the treatment of skin cancer. Its chief application may be skin lesions in locations difficult for surgical dressing, large lesions and patients with numerous concomitant diseases. Imiquimod treatment guarantees a good cosmetic effect, high oncological efficacy and the possibility for patients to apply the preparation themselves. Before beginning the therapy it is necessary to inform the patient of the possibility of occurrence of an intensified inflammatory reaction around the lesion. Further multi-centre research with longer patient observation time is necessary, especially in order to evaluate long-term results of the therapy.

\section{References}

1. Mikkilineni R, Weinstock MA. Epidemiology of skin cancer. Atlas of clinical oncology: skin cancer. London UK:BC Decker, Inc., 2001; 1-15.

2. Schoen M, Schoen MP. The antitumoral mode of action of imiquimod and other imidazoquinolines. Curr Med Chem 2007; 14: 681-687.

3. Schoen $M$, Bong AB, Drewniok C et al. Tumor- selective induction of apoptosis and the small molecule immune response modifier imiquimod. J Natl Cancer Inst 2003; 95: 1138-49.

4. Li VW, Li WW, Talcott KE, et al. Imiqumod as an antiangiogenic agent. J Drugs Dermatol 2005; 4: 708-16.

5. Wagstaff AJ, Perry CM. Topical imiquimod -a review of its use in the management of anogenital warts, actinic keratoses, basal cell carcinoma and other skin lesions. Drugs 2007; 67: 2187-2210.

6. Nguyen T, Ho D. Nonmelanoma skin cancer. Curr Treat Options Oncol 2002;3:193-203.

7. Marks R, Gebauer K, Shumack S, et al. Imiquimod 5\% cream in the treatment of superficial basal cell carcinoma: results of a multicenter 6 week dose-response trial. J Am Acad Dermatol 2001;44:807-813.

8. Geisse JK, Rich P, Pandya A, et al. Imiquimod 5\% cream in the treat ment of superficial basal cell carcinoma: a double-blind, random ized vehicle control study. J Am Acad Dermatol 2002;47:390-398.

9. Peris K, Campione E, Micantonio T, Maruli GC, Fargnoli MC, Chimenti S. Imiquimod treatment of superficial and nodular basal cell carcinoma: 12-week open label trial.Dermatol Surg 2005; 31: 318 323.

10. Hanger C, Dalrymple J, Hepburn D. Systemic side effects from topical imiquimod. N Z Med I 2005; 118: U1682.

11. Schulze HJ, Cribier B, Requena L, et al. Imiquimod 5\% cream for the treatment of superficial basal cell carcinoma: results from a randomized vehicle-controlled phase III study in Europe. Br J Dermatol 2005; 152: 939-947.
12. Torres A, Niemeyer A, Berkes B, et al. Treatment of basal cell carcinoma using imiquimod 5\% cream as an adjuvant therapy to Mohs micrographic surgery. J Eur Acad Dermatol Venereol 2003; 17 (Suppl 3).

13. Spencer JM, Pilot study of Imiquimod 5\% cream as an adjunctive therapy to curettage and electrodessication for nodular basal cell carcinoma. Dermatol Surg 2006; 32: 63-69.

\section{Address for correspondence}

Dawid Murawa MD, PhD

Greater Poland Cancer Center

ul. Garbary 15

61-866 Poznań

e-mail: dmurawa@op.p 